Reprod. Nutr. Dévelop., 1988, 28 (1), 191-192.

\title{
Particularités de l'utilisation des substrats glucoformateurs par les hépatocytes isolés de mouton
}

C. YACOUB, C. REMÉSY, C. DEMIGNÉ

Laboratoire des Maladies Métaboliques, I.N.R.A., Theix, 63122 Ceyrat, France.

Summary. Gluconeogenesis from various substrates was compared in rat and sheep hepatocytes. Lactate, alanine and glycerol were important precursors in the rat but poorly glucogenic in sheep hepatocytes, for which propionate was the main glucogenic substrate. Glutamine utilization was more efficient with sheep hepatocytes and is inhibited by acidosis, but unlike rat, it was not stimulated by ammonia.

A la différence du monogastrique, la néoglucogenèse chez le ruminant est maximale à l'état nourri grâce à la forte disponibilité en propionate. Durant le jeûne, des substrats tels que le glycérol et les acides aminés peuvent se substituer au propionate. Toutefois, excepté le propionate, l'utilisation hépatique des substrats glucogéniques semble peu active (Naylor et al., 1984). L'utilisation vers la néoglucogenèse des différents précurseurs a donc été réexaminée sur hépatocytes isolés de ruminant (mouton), en procédant à une comparaison avec un monogastrique (rat).

Matériel et méthodes. L'isolement d'hépatocytes de mouton à jeun depuis $24 \mathrm{~h}$ a été effectué juste après l'abattage. Un morceau d'environ $50 \mathrm{~g}$ de foie est perfusé sur place, via une branche portale, par du tampon Krebs-bicarbonate (KB) sans $\mathrm{Ca}^{++}$, puis au laboratoire par un tampon $\mathrm{KB}$ contenant $2 \mathrm{mM}$ de $\mathrm{Ca}^{++}$et $\approx 0,5 \mathrm{mg} / \mathrm{ml}$ de collagénase. Au bout de $30 \mathrm{~min}$, les hépatocytes sont recueillis, filtrés et rincés à 3 reprises dans du tampon KB contenant $2 \%$ d'albumine (Demigné et al., 1986). L'isolement des hépatocytes de rat est effectué selon une technique très semblable. Les cellules $(\approx 25 \mathrm{mg} / \mathrm{ml})$ ont été incubées $30 \mathrm{~min}$, en présence des divers substrats.

Résultats et discussion. La conversion en glucose des divers précurseurs (à $2,5 \mathrm{mM}$ ) est très élevée chez le rat, excepté pour la glutamine et dans une certaine mesure pour l'alanine (fig. 1). Par contre, chez le mouton, le propionate se distingue nettement des autres substrats, dihydroxyacétone et fructose mis à part. L'utilisation du lactate et de l'alanine est faible, alors que celle de la glutamine est plus élevée que chez le rat. Chez le mouton, le lactate est moins glucogénique que le pyruvate, à l'inverse du rat, il faut noter que, à $2,5 \mathrm{mM}, 30 \% \mathrm{du}$ pyruvate est transformé en lactate chez le rat contre seulement $25 \%$ chez le mouton. A cet égard, il reste à déterminer la rapidité du transport du pyruvate dans les mitochondries, et l'activité effective de la pyruvate carboxylase mitochondriale; on sait en effet que ces deux étapes sont particulièrement activées chez le rat. Lomax et al. (1986), sur hépatocytes de mouton à jeun 4 jours, ont obtenu une néoglucogenèse notable à partir de lactate ou pyruvate $140 \%$ de celle à partir du propionate). Toutefois, ces auteurs ont utilisé des concentrations très élevées $(10 \mathrm{mM})$ en substrats. L'utilisation basale de la glutamine (GN) par les hépatocytes est plus forte chez le mouton que chez le rat (tabl. 1) ; elle est égale à celle de l'alanine pourtant considérée comme le principal acide aminé glucoformateur. $\mathrm{NH}_{3}$, le plus puissant effecteur de la glutaminase chez le rat, modifie peu, 
I'utilisation de la glutamine chez le mouton. Par contre (fig. 2), la baisse du pH et du $\mathrm{HCO}_{3}$-extracellulaire diminue sensiblement l'utilisation de la GN par les hépatocytes de mouton (de même que chez le rat) ainsi que l'uréogenèse globale. Cette dépression de l'activité glutaminasique, connue in vivo (Heitmann et Bergmann, 1980), pourrait refléter la déplétion en bicarbonate ou en phosphate libre mitochondrial.

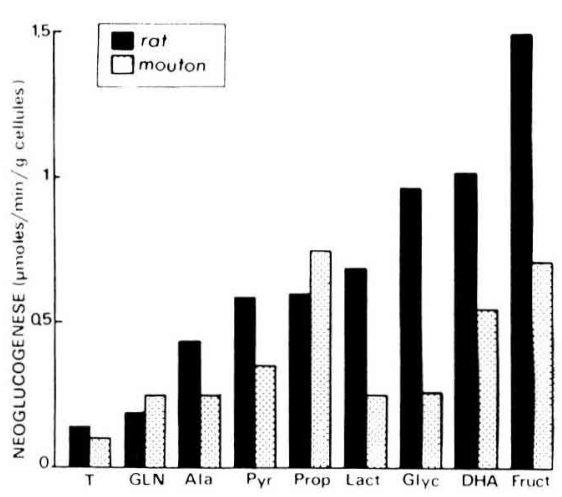

FIG. 1. - Comparaison de l'utilisation des différents précurseurs glucoformateurs $(2,5 \mathrm{mM})$. Témoin (T), glutamine (GLN), alanine (Ala), pyruvate (Pyr), propionate (Prop), lactate (Lact), glycérol (Glyc), dihydroxyacétone (DHA) et fructose (Fruct).

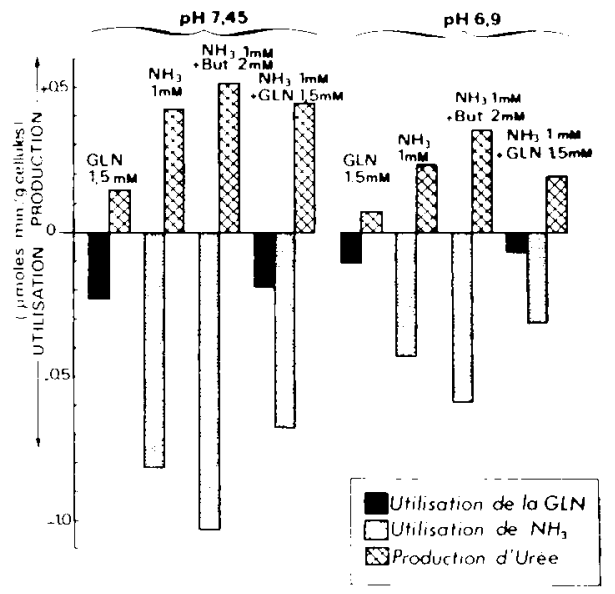

FIG. 2. - Effet de l'acidose extracellulaire ([ $\left.\left.\mathrm{HCO}_{3}-\right] 10 \mathrm{mM}, \mathrm{pH} 6,9\right)$ sur I'utilisation de la g/utamine et de $\mathrm{NH}_{3}$ et sur l'uréogenèse. GLN : glutamine ; But : butyrate.

TABL. 1. - Comparaison de l'utilisation de la g/utamine par des hépatocytes isolés de rat et de mouton ( $\mu$ moles $/ \mathrm{mn} / \mathrm{g}$ poids frais \pm SEM pour 5 lots d'hépatocytes).

\begin{tabular}{lccccc} 
& \multicolumn{2}{c}{ Mouton } & \multicolumn{2}{c}{ Rat } \\
\cline { 2 - 5 } & Utilisation & Uréogenèse & Utilisation & Uréogenèse \\
\hline Glutamine 2,5 mM & $\begin{array}{c}0,27 \pm 0,03 \\
\text { Glutamine 2,5 mM }+\mathrm{NH}_{3} 1 \mathrm{mM}\end{array}$ & $\begin{array}{c}0,21 \pm 0,02 \\
0,25 \pm 0,03\end{array}$ & $\begin{array}{c}0,14 \pm 0,02^{*} \\
\left.\text { (dont } \mathrm{NH}_{3} 0,20\right)\end{array}$ & $\begin{array}{c}0,18 \pm 0,02 \\
0,05 \pm 0,07^{*}\end{array}$ & $\begin{array}{c}1,05 \pm 0,10^{*} \\
\left(\text { dont } \mathrm{NH}_{3} 0,55\right\}\end{array}$ \\
\hline
\end{tabular}

* Différence significative entre les deux espèces $(P<0,05)$.

En conclusion, les modalités de la néoglucogenèse chez le mouton apparaissent distinctes de celles rapportées chez le monogastrique. Les substrats dont le métabolisme initial s'effectue dans les mitochondries sont efficacement utilisés vers la néoglucogenèse, ce qui est à rapprocher de l'existence d'une phosphoénolpyruvate carboxykinase mitochondriale. Par contre, à la différence du rat, les substrats qui aboutissent à l'apparition de pyruvate dans le cytoplasme sont peu efficaces.

Demigné C., Yacoub C., Rémésy C., Fafournoux P., 1986. Biochim. Biophys. Acta, 875, $535-542$. Naylor J. M., Kronfeld D. S., Freeman D. E., Richardson D., 1984. Am. J. Physiol, 247, E747-E755. Heitmann R. N., Bergman E. N., 1980. Am. J. Physiol., 239, E248-E254.

Lomax M. A., Donaldson I. A., Pogson C. I., 1986. Biochem. J., 240, 277-280. 